\title{
Coordination and Compensation Model of E-Commerce Dual-Channel Supply Chain Based on Optimized Genetic Algorithm
}

\author{
Wei Liu \\ Wuhan Technical College of Communications, Wuhan 430065, China \\ E-mail:1w554776880@163.com
}

Received: June 3, 2021. Revised: December 19, 2021. Accepted: January 11, 2022. Published: January 13, 2022.

\begin{abstract}
Due to the conflict between traditional channels and electronic channels in the e-commerce dual-channel supply chain, retailers are threatened and need to be compensated in some way. Based on this, an e-commerce dual-channel supply chain coordination compensation model based on optimized genetic algorithm is designed. Based on the problem description and basic assumptions, analyze the manufacturer's profit and the retailer's maximum profit in the case of centralized decision-making and decentralized decision-making. The genetic algorithm is optimized by introducing a collaborative genetic operator, and the optimized genetic algorithm is used to obtain dual e-commerce channels. The maximum profit of the supply chain, so far, the model design is completed. Through comparative experiments, the optimized genetic algorithm used in the model is compared with two traditional algorithms. Experimental results show that the proposed algorithm takes shorter iteration time to solve the problem, its convergence is better, and it can effectively obtain a global optimal solution instead of a local optimal solution.
\end{abstract}

Keywords - dual-channel supply chain, supply chain coordination, collaboration operator, compensation strategy.

\section{INTRODUCTION}

$\mathrm{I}^{\mathrm{N}}$ $\mathrm{N}$ the era of e-commerce, whether from the perspective of improving the market competitiveness of enterprises or from the perspective of customer demand, the implementation of a dual-channel strategy will be an inevitable trend for manufacturers to conform to the development of the times. At present, some large companies have successfully applied the dual-channel strategy to actual business operations. Balancing the relationship between electronic channels and traditional channels through effective means can not only greatly improve market penetration, but also bring about a new value transfer model [1]-[4]. However, because many traditional retailers regard the electronic direct sales channels opened by the manufacturers as a threat, they are hostile or even resistant to the manufacturer's move, which makes the conflict between the members of the dual-channel supply chain become more and more. Highlight [5]-[7]. In the traditional channel supply chain environment, in order to alleviate or eliminate the double marginalization problem caused by the externalities of the upstream and downstream members of the channel, relevant scholars have proposed corresponding coordination mechanisms [8], [9]. However, in the dual-channel supply chain, the manufacturer is both a supplier of the retailer and a competitor of the retailer. This leads to the existence of both upstream and downstream dual marginalization problems in the dual-channel supply chain, as well as channels of the same level. Conflict makes the general supply chain contract unable to coordinate the dual-channel supply chain of a single manufacturer and a single retailer. Therefore, in the e-commerce environment, how to achieve the coordination of the dual-channel supply chain, give play to the advantages of dual-channel, increase profits and reduce inventory is a new problem in theoretical research and practical management [10]-[12]. At present, related scholars have done some research on the coordination and compensation of the dual-channel supply chain of e-commerce. For example, the coordinated compensation model based on random targets [13] and the coordinated compensation model based on environmental factors [14], but these models still have certain shortcomings and need to be further studied. The genetic algorithm seeks the optimal solution through continuous iterations to solve the problem [15]. This algorithm has many advantages such as fast optimization speed, simple process and compatibility with other algorithms. Pramono et al. [16] presented the impact of entrepreneurial capability, agility, business transformation and opportunities on the entrepreneurial behavior of Indonesia e-commerce company in COVID-19. Chernova and Degtereva [17] presented the development forecast of the industrial dual-use product market, and Eldar [18] put forward the issue of gold fusion investment support model for regional social and economic development. Based on this, an e-commerce dual-channel supply chain coordination compensation model based on optimized genetic algorithm is designed. Use this model to realize the coordinated compensation for the dual-channel supply chain of 
e-commerce.

\section{E-COMMERCE DUAL-CHANNEL SUPPLY CHAIN COORDINATION COMPENSATION MODEL BASED ON OPTIMIZED GENETIC ALGORITHM}

\section{A. E-commerce Dual-channel Supply Chain} Coordination Compensation Model

Simplify the channel model to the coordination problem of a dual-channel supply chain composed of a manufacturer and a retailer, where both the manufacturer and the retailer are risk-neutral and completely rational, that is, both will be based on the principle of maximizing expected profits to make decisions. The manufacturer takes the lead and the retailer takes the lead. Manufacturers use traditional channels to distribute products with a unit production cost of $z$ to retailers at the wholesale price $l$. At the same time, they open up new electronic channels to sell directly to consumers at the price $p_{e}$. At this time, the retailer, based on the wholesale price and the electronic direct sales price, it is sold to consumers at a price $p_{r}$ in traditional retail channels. Based on the above, suppose the demand functions of traditional retail channels and electronic direct sales channels are respectively:

$$
\begin{aligned}
& v_{r}=w o-p_{r}+u p_{e} \\
& v_{e}=(1-w) o-p_{e}+u p_{r}
\end{aligned}
$$

In Formula (1), $v_{r}$ represents traditional retail channel demand, $v_{e}$ represents electronic direct sales channel demand, $o(o>0)$ represents total market demand, $w(0<w<1)$ represents the market share of traditional channel retailers under dual channels, and $u(0<u<1)$ represents the gap between the two channels The cross-price elasticity coefficient is required to be $w o-p_{1}>0$ and $(1-w) o-p_{2}>0$, which means that there are loyal customers in both retail channels and electronic channels. A dual channel supply chain coordination problem composed of a manufacturer and a retailer, in which both manufacturers and retailers are risk neutral and completely rational, that is, they will make decisions according to the principle of maximizing expected profits. The manufacturer is the main party and the retailer is the subordinate party. On this basis, determine the form of decision-making.

\section{B. Determination of the Form of Decision-Making}

In the context of centralized decision-making in the dual-channel supply chain of e-commerce, assuming that traditional retail channels are also owned or controlled by manufacturers with direct e-commerce channels, they will jointly pursue the maximum profit of the entire dual-channel supply chain. Manufacturers and retailers will form a unified whole, which is an ideal decision-making method. Its purpose is to improve the interests of the overall supply chain through power concentration. It is widely used in reality. This power concentration can be realized by the cooperation and coordination of each node enterprise in the supply chain. In this case, the decision made is globally optimal, and provides a benchmark for the coordination research of the dual-channel supply chain under decentralized decision-making. Therefore, the profit function of the entire dual-channel supply chain in the case of centralized decision-making is:

$$
\begin{aligned}
& \sigma_{I}=\left(p_{r}-z\right)\left(w o-p_{r}+u p_{e}\right)+ \\
& \left(p_{e}-z\right)(1-w) o-p_{e}+u p_{r}
\end{aligned}
$$

In Formula (3), $\sigma_{I}$ represents the profit function of the entire dual-channel supply chain under the centralized decision-making situation. From Formula (3), it can be seen that the profit function of the entire dual-channel supply chain is a binary concave function of the traditional retail price and the electronic direct sales price. Further processing can obtain the optimal electronic direct sales price and the best traditional centralized decision-making the retail price, substituting it into the demand function for further processing, the profit of the dual-channel supply chain under centralized decision-making can be obtained as:

$$
\begin{array}{r}
\left(\frac{1}{2}-(1-w)(1-u) w u\right) o^{2}- \\
\sigma_{I}^{\prime}=\frac{\left(1-u^{2}\right) z o+\left(u^{3}-u+1\right) z^{2}}{2\left(1-u^{2}\right)}
\end{array}
$$

In the case of decentralized decision-making in the dual-channel supply chain, the manufacturer and the retailer make independent decisions to maximize their profits. The manufacturer's profit function and the retailer's profit function are respectively:

$$
\begin{aligned}
& \sigma_{m}=(l-z)\left(w o-p_{r}+u p_{e}\right)+ \\
& \left(p_{e}-z\right)\left((1-w) o-p_{e}+u p_{r}\right) \\
& \sigma_{r}=\left(p_{r}-l\right)\left(w o-p_{r}+u p_{e}\right)
\end{aligned}
$$

Using the principle of master-slave strategy to solve Formula (5) and Formula (6), we can get the best electronic direct selling price can be get, the best wholesale price and the best traditional retail price, substituting them into the demand function and further processing can obtain the profits of manufacturers, retailers and dual-channel supply chains under decentralized decision-making, and processing it, the difference between the profits of centralized decision-making and decentralized decision-making can be obtained as the Formula (7) shown:

$$
\Delta \sigma^{\prime}=\sigma_{I}{ }^{\prime}-\left(\sigma_{m}{ }^{\prime}+\sigma_{r}{ }^{\prime}\right)=\frac{(w o+u z-z)^{2}}{16}
$$

Calculate the profit difference between the two decision-making forms by Formula (7). In a dual-channel supply chain, when the decentralized decision-making is consistent with the optimal decision under the centralized decision-making, it will be able to achieve dual-channel supply chain coordination. Through calculation, it can be seen that the optimal traditional retail price under decentralized decision-making of dual channel supply chain is greater than that under centralized decision-making, and it is found that the supply chain profit under centralized 
decision-making is higher than that under decentralized decision-making. Significant increase (the increased profit here is equal to the retailer's profit under the decentralized decision), that is, the dual channel supply chain under the decentralized decision can not reach the coordination state. Therefore, in the manufacturer led dual channel market, how to encourage retailers to continue to participate in traditional retail channels and effectively coordinate traditional retail channels and e-Direct sales channels, so as to improve the overall profit of the supply chain and obtain the optimal value of the overall profit is the manufacturer's goal. In the dual channel market dominated by the manufacturer, in order to alleviate the possible channel conflict and realize the coordination of the dual channel supply chain, the manufacturer promises to provide a certain proportion of electronic channel orders to retailers as compensation to encourage retailers to continue their cooperation. At the same time, in order to ensure that their own interests are not damaged, the manufacturer will charge a certain "license fee" from retailers as a threshold for compensation. Under this compensation strategy, by setting different compensation coefficients and calculating the profit function, the profit of the dual-channel supply chain can be obtained as shown in Formula (8):

$$
\begin{array}{r}
\left(\frac{1}{2}-(1-w)(1-u) w u\right) o^{2}- \\
\sigma_{I}{ }^{\prime}=\frac{\left(1-u^{2}\right) z o+\left(u^{3}-u+1\right) z^{2}}{2\left(1-u^{2}\right)}
\end{array}
$$

In Formula (8), the profit of the coordinated dual-channel supply chain is the sum of the manufacturer's profit and the retailer's profit. Manufacturers can encourage retailers to cooperate through the above compensation strategy, so that the dual-channel supply chain can reach a coordinated state, that is, the total profit of the dual-channel supply chain can be maximized. However, due to the constraints of individual rationality, supply chain members first consider their own interests. For the manufacturer, the manufacturer will only provide the above compensation strategy when the profit after coordination is greater than the profit before the coordination; for the retailer, only when the profit after coordination is greater than the profit before the coordination, the retailer is willing to accept this kind of compensation. In other words, it is necessary to ensure that the profit of each member under this compensation strategy is improved, that is, only when the supply chain members are guaranteed a win-win situation, can the manufacturer be willing to provide and the retailer is willing to accept this compensation strategy, in order to be able to effectively coordinate the dual-channel supply chain. The established dual-channel supply chain coordination compensation model is based on the offline retail network and the online direct sales network, which includes multiple offline nodes and multiple online nodes. Therefore, the optimized genetic algorithm is selected to solve the problem. The detailed analysis will be made below.

\section{Optimal Selection of Genetic Algorithm}

(1) Algorithm optimization
The improvement of the selection strategy is mainly divided into two parts: Firstly, the individual optimal value of the parent is retained during the selection phase, and the optimal value is taken as a solution in the solution set. Secondly, in the later stage of population evolution, the fitness values of excellent individuals are prioritized to make excellent individuals more competitive than ordinary individuals [19]. Crossover and mutation improvement: Due to the complexity of the determination process, it is usually selected as a fixed value based on experience. Therefore, when facing different optimization problems, the solution rate will decrease. Through the adaptive genetic algorithm that optimizes the crossover probability $P_{c}$ and the mutation probability $P_{m}$, the crossover probability can be expressed by Formula (9):

$$
P_{c}= \begin{cases}P_{c 1}-\frac{\left(P_{c 1}-P_{c 2}\right)\left(t-t_{\text {avg }}\right)}{t_{\max }-t_{\text {avg }}} & t \geq t_{\text {avg }} \\ P_{c 1} & t<t_{\text {avg }}\end{cases}
$$

In Formula (9), $t_{\max }$ represents the maximum fitness, $t_{\text {avg }}$ represents the average fitness, $t$ represents the fitness of the outstanding individuals to be crossed, $P_{c 1}$ represents the maximum crossover probability, and $P_{c 2}$ represents the minimum crossover probability. The probability of mutation is expressed by Formula (10):

$$
P_{m}= \begin{cases}P_{m 1}-\frac{\left(P_{m 1}-P_{m 2}\right)\left(t-t_{\text {avg }}\right)}{t_{\text {max }}-t_{\text {avg }}} & t \geq t_{\text {avg }} \\ P_{m 1} & t<t_{\text {avg }}\end{cases}
$$

In Formula (10), $P_{m 1}$ represents the probability of high frequency mutation, and $P_{m 2}$ represents the probability of low frequency mutation. After selecting the algorithm, carry out algorithm analysis. On the basis of the standard genetic algorithm, the collaborative genetic operator (cooperative operator and swallowing operator) is introduced to optimize the algorithm [20]. The specific analysis process is described in the following content. Among them, the design of the cooperation operator is as follows: in the algorithm initialization stage, two populations $\quad q^{a}{ }_{t}=\left(e^{a}{ }_{1}, e^{a}{ }_{2}, \cdots, e^{a}{ }_{M}\right) \quad$ and $q^{b}{ }_{t}=\left(e^{b}, e_{2}^{b}, \cdots, e^{b}{ }_{N}\right)$ are set, $\left[R_{t}^{a}, R_{t}^{b}\right]$ is the optimal solution set of the population, and any individual $\left(d^{a}{ }_{1}, d^{a}{ }_{2}, \cdots, d^{a}{ }_{M}\right) \quad$ in $\quad q^{a}{ }_{t}$ and a individual $\left(s_{1}^{b}, s_{2}^{b}, \cdots, s_{n}^{b}\right)$ randomly selected from $U_{t}^{b}$ according to Formula (11) to generate a new individual $\left(e^{a}{ }_{1}, e^{a}{ }_{2}, \cdots, e^{a}{ }_{n}\right)$ to form a new population $q^{a}{ }_{t+1}$ :

$$
e_{i}^{a}=s_{i}^{a}+U(-1,1) \times\left(s_{i}^{b}-d_{i}^{a}\right) i=1,2, \cdots, n
$$

In Formula (11), $U(-1,1)$ is uniformly distributed between $(-1,1)$. Similarly, for any individual $\left(d^{b}, d^{b}{ }_{2}, \cdots, d^{b}{ }_{M}\right)$ in $q^{b}{ }_{t}$ in the initial population, and 
any solution $\left(s_{1}^{b}, s_{2}^{b}, \cdots, s_{n}^{b}\right)$ in $R_{t}^{a}$, a new individual $\left(e^{b}{ }_{1}, e^{b}{ }_{2}, \cdots, e^{b}{ }_{n}\right)$ is generated according to Formula (12), and a new population $q_{t+1}^{b}$ is formed:

$$
e_{i}^{a}=s_{i}^{a}+U(-1,1) \times\left(s_{i}^{b}-d_{i}^{a}\right) i=1,2, \cdots, n
$$

The design process of the annexation operator is as follows: Assuming that the initial populations $q^{a}{ }_{t}=\left(e^{a}{ }_{1}, e^{a}{ }_{2}, \cdots, e^{a}{ }_{M}\right)$ and $q^{b}{ }_{t}=\left(e^{b}{ }_{1}, e^{b}{ }_{2}, \cdots, e^{b}{ }_{N}\right)$ have optimal solutions $R_{t}^{a}$ and $R_{t}^{b}$, if the following relationship exists:

$$
\forall d \in R_{t}^{a}, \exists e \in R_{t}^{b}, d<z
$$

At this time, population $b$ has swallowed population $a$ and formed a new progeny population $q^{a}{ }_{t+1}=\left(k_{1}, k_{1}, \cdots, k_{N+M}\right) \quad$. In the formula, $k_{i}=e_{i}, i=1,2, \cdots, N \quad$ and $\quad k_{i}(i=N+1, N+2, \cdots, N+M)$ are produced by Formula (14):

$$
k_{i j}=s_{j}+U(-1,1) \times\left(s_{j}-d_{i-N, j}\right) j=1,2, \cdots, n
$$

The solution set $R_{t}^{b}$ of b population contains solutions of $R_{t}^{a}$, that is, the performance of population $b$ is better than that of population $a$. The probability of obtaining excellent solutions of population $a$ is lower than that of population $b$. Eliminate population $a$ and keep the excellent individuals of population $a$. Simplify calculations. Divide $q_{t}=\left(d_{1}, d_{2}, d_{3}, \cdots, d_{m}\right)$ into two progeny populations $q^{a}{ }_{t}=\left(e^{a}{ }_{1}, e^{a}{ }_{2}, \cdots, e^{a}{ }_{M / 2}\right) \quad$ and $\quad q^{b}{ }_{t+1}=\left(e^{b}{ }_{1}, e^{b}{ }_{2}, \cdots, e^{b}{ }_{M / 2}\right)$ randomly, and make a mutation operation as shown in Formula (15) for a progeny population and all individuals of $q^{a}{ }_{t+1}$ :

$$
e_{i}^{a}=\left\{\begin{array}{lc}
e_{i}^{a} & U(0,1)<1 / n \\
e_{i}^{a}+G(0,1 / \alpha) & U(0,1) \geq 1 / n
\end{array}\right\}
$$

In Formula (15), $G$ represents Gaussian distribution, and $\alpha$ represents chromosome evolution algebra. The optimized genetic algorithm is based on the simultaneous evolution and cooperation of multiple populations to carry out information exchange, and there is a competitive relationship in the process of cooperation [21]. The population with poor solution quality is eliminated by the annexation operator; when the population is too large, after a certain iteration, the population diversity decreases. At this time, the split operator is used to generate a new population, and the diversity of the population is maintained until a solution set that meets the requirements is obtained.

(2) Obtaining the optimal solution

The specific steps of the algorithm are as follows: The first step is to set two populations $a$ and $b$ with a scale of $N$. $R^{a}$ and $R^{b}$ are the optimal solution sets of the populations $a$ and $b$, respectively, and $\bar{Q}$ represents the out-of-population set, and its scale is $\bar{N}$. In the second step, the population is initialized and calculated in parallel to obtain two optimal solution sets $R^{a}$ and $R^{b}$, and set $\bar{Q} \leftarrow \varphi, t \leftarrow 0$ at the same time. The third step is to update the outer set $\bar{Q}_{t}$ with $R^{a}$ and $R^{b}$ to obtain a new set $\bar{Q}_{t+1}$. In the fourth step, $\bar{Q}_{t}$ is divided into two parts $Q_{t}{ }^{a}$ and ${ }^{-}{ }^{b}$, and the optimal individual retention strategy is executed for the two populations at the same time, and a new set $Q_{t}{ }^{1 a}$ and $\bar{Q}_{t}{ }^{1 b}$ is formed under the retention strategy. The fifth step is to determine whether $\bar{Q}_{t}{ }^{1 a}$ and $\bar{Q}_{t}{ }^{1 b}$ meet the termination conditions. If they are satisfied, the algorithm terminates and outputs $\bar{Q}_{t+1}$. If not, expand $\bar{Q}_{t}{ }^{1 a}$ and $\bar{Q}_{t}{ }^{1 b}$ according to the mutation probability $Q_{m}$, and add the co-evolution operator. In the sixth step, cross $Q_{t}{ }^{1 a}$ and $Q_{t}^{-1 b}$ to get $Q_{t+1}^{-1 a}$ and $Q_{t+1}^{-{ }^{1 b}}$. If $\bar{R}_{t+1}^{n a}$ is included in $\bar{R}_{t+1}^{n b}$, then execute the annexation operation of $Q_{t+1}^{-1 a}$ to $Q_{t+1}^{-1 b}$; otherwise, execute the cooperative operator operation to obtain $Q_{t+2}^{-1 a}$ and $Q_{t+2}^{-}{ }^{1 b}$. The seventh step is to evaluate the fitness. If the termination condition is met, $Q_{t+2}^{-}$is output. If it is not satisfied, return to the second step [22]. Among them, when generating the initial population, the solution of the dual-channel supply chain coordination and compensation problem is understood as a chromosome, which is specifically expressed in the algorithm as a specially coded string. When performing calculations, first randomly generate an initial population based on the constraints of the problem, and each individual in the population can essentially be regarded as a genetically encoded chromosome, in which the optimal solution to the decision-making problem may be contained in it. The chromosome coding requirements in the initial population cannot be repeated, and the 0 and 1 in the chromosome coding position of each individual are randomly generated with equal probability [23], [24]. The individual generator randomly generates 0 and 1 code strings to record the individuals that have been generated. If the same individual is generated by randomness, the random generator is required to regenerate until it generates an individual that is different from the existing individual. After encoding, the individual is decoded and fitness calculation is performed. When solving, it is necessary to link the concept of the solution of the target problem with the concept of the chromosome string in the genetic algorithm. Therefore, it is necessary to convert the solution space of the problem to the search space that the genetic algorithm can handle through coding. Use binary coding to encode it. Firstly, determine the range of the selected sales price ratio, then 
determine the number of binary coding digits, accurate to 4 digits after the decimal point, and finally, determine the mapping relationship between binary numbers and real numbers. Combining the characteristics of the dual-channel supply chain and the coordination compensation model, using optimized genetic algorithms, the selection strategy and genetic operators are improved, and the collaborative operator is introduced to determine the state of the optimal solution set and perform the operations of annexation, division, and cooperation. For the existing competition and cooperative relations in the supply chain, simulate the behavior of annexation or cooperation, so as to obtain a better solution set until the optimal solution is found. So far, the e-commerce dual-channel supply chain coordination compensation model based on the optimized genetic algorithm has been established.

\section{3 EXPERIMENT}

\section{A. Case Background}

Take a certain company as a model case. The company is a manufacturing-sales company with chain stores in many cities in China and a sales network all over the country. This example uses the company's supply chain network in $\mathrm{H}$ City in the Northeast as a background to establish a dual-channel supply chain model. Mathematica and MATLAB are used to calculate the explicit solutions of decision variables and profits, compare the strategy differences under different decision modes, and draw the impact trend diagram of random demand on pricing, inventory and profits to reveal the management enlightenment.

\section{B. Experimental Process}

Taking the profit of the dual-channel supply chain of e-commerce as the goal, the optimization genetic algorithm is used to solve the problem. The parameter settings of the optimization algorithm are shown in Table 1.
Table 1. Optimization of genetic algorithm parameter settings

\begin{tabular}{ccc}
\hline $\begin{array}{c}\text { Serial } \\
\text { number }\end{array}$ & Parameter name & Parameter value \\
\hline 1 & Population size & 200 \\
2 & $\begin{array}{c}\text { Initial crossover } \\
\text { probability } \\
\text { Initial mutation } \\
\text { probability }\end{array}$ & 250 \\
4 & $\begin{array}{c}\text { Maximum number of } \\
\text { iterations }\end{array}$ & 0.8 \\
\hline
\end{tabular}

By using the above parameters and using optimized genetic algorithm, the optimal solution of profit maximization of e-commerce supply chain is obtained through iteration. Set different compensation parameters, compare the speed of the optimized genetic algorithm and the two traditional algorithms to obtain the optimal solution, compare the iterative termination time of the three algorithms to obtain the optimal solution (the maximum profit of the e-commerce supply chain). The specific experimental results are shown below.

\section{Experimental Results}

Using the proposed e-commerce dual-channel supply chain coordination compensation model based on optimized genetic algorithm, the traditional e-commerce channel supply chain coordination compensation model 1 , the traditional e-commerce channel supply chain coordination compensation model 2 , and the algorithm used to find the optimal solution, the comparison result of the iteration termination time is shown in Figure 1.

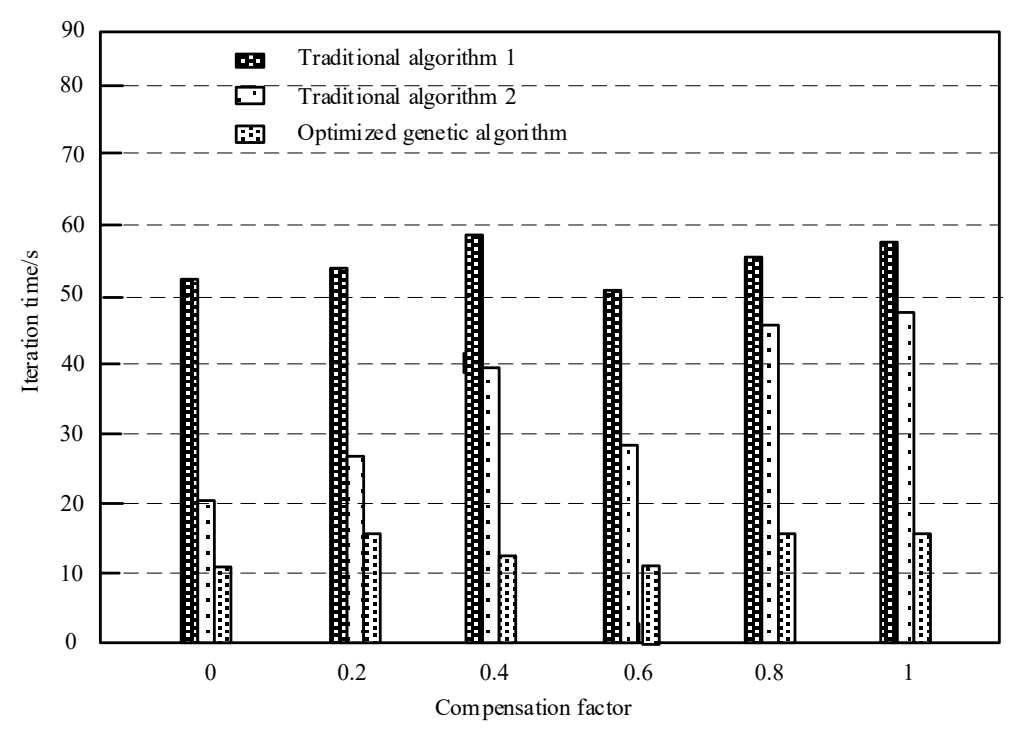

Figure 1. Comparison results of iteration termination time

It can be seen from Figure 1 that using traditional algorithm 1, its iteration time is more than $50 \mathrm{~s}$; using traditional algorithm 2, its iteration time ranges from 20 to $50 \mathrm{~s}$; using the proposed optimized genetic algorithm, its iteration time is $10-15 \mathrm{~s}$. Through comparison, it is found that the proposed optimization genetic algorithm has shorter iteration time and better convergence when solving the problem. On this basis, the maximum profit comparison 
results of the e-commerce dual-channel supply chain obtained by using the three algorithms are shown in Figure

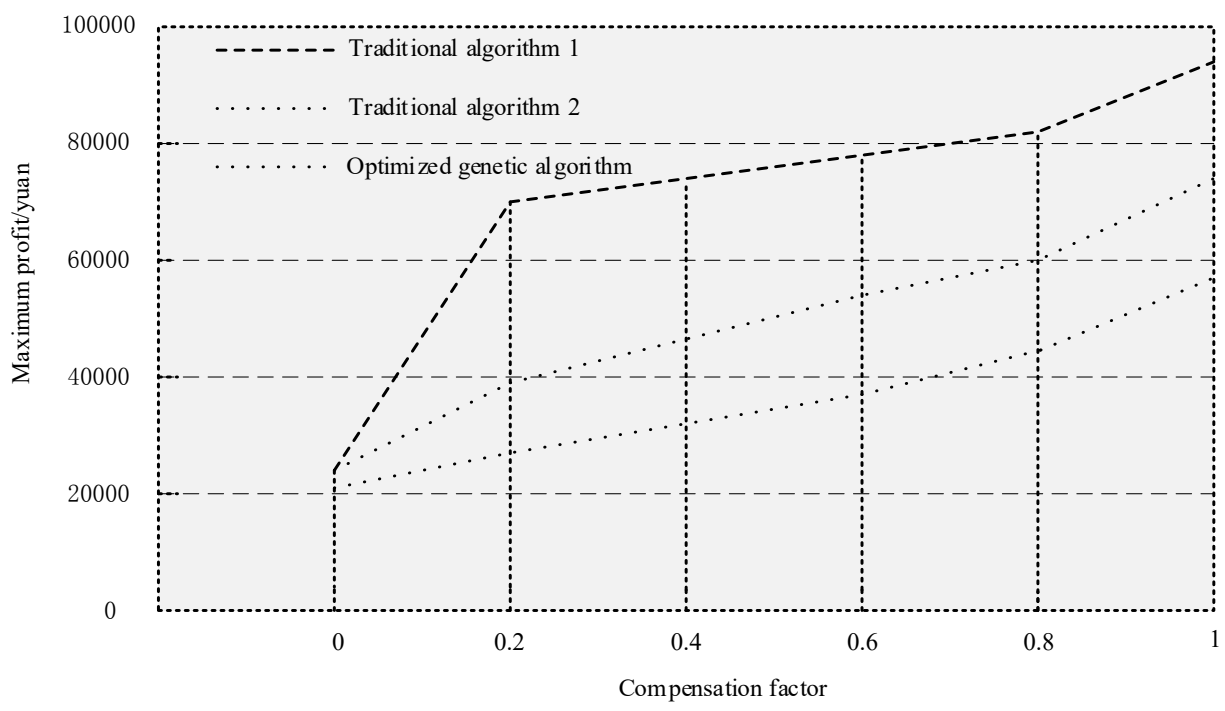

Figure 2. Comparison results of maximum profit

By comparing the maximum profit in Figure 2, it can be seen that the maximum profit obtained by the proposed optimization genetic algorithm is the global optimal solution. Taking the compensation coefficient of 1 as an example, the maximum profit can reach up to RMB 93,985, while the maximum profits of the other two traditional algorithms are 74,126 yuan and 57,793 yuan, respectively. From the perspective of supply chain profit, the dominant party has the largest profit regardless of whether the manufacturer is dominant or the retailer is dominant, which indicates that supply chain members can seek greater profit for themselves by virtue of dominant power. In the case of equal decision-making status, the profit is the smallest. First, because the product pricing of both sides of the game is low, the market demand and inventory are small, and the profit is small. The double marginal effect of the two is to reduce the individual income. The overall profit level of the manufacturer-led supply chain is higher than that of the retailer-led supply chain, because manufacturers control network channels and physical channels and have stronger channel integration ability. Through comparison, it is found that the proposed optimization genetic algorithm will fall into a local optimal solution, and the optimal solution can be obtained effectively.

\section{4 CONCLUSIONS}

(1) In view of the dual channel model of the coexistence of traditional retail channels and e-Direct sales channels in e-commerce environment, through the comparative analysis of the optimal price and profit changes of dual channel supply chain under centralized and decentralized decision-making, it is found that traditional channels and e-channels are competitive due to price, which will lead to channel conflict.

(2) In order to alleviate the channel conflict and make the dual channel supply chain reach the coordination state, through the research on this compensation strategy mechanism, we can realize the coordination of the dual channel supply chain and realize the win-win of the members of the dual channel supply chain. Aiming at the shortcomings of the traditional e-commerce dual channel supply chain coordination and compensation model, an e-commerce dual channel supply chain coordination and compensation model based on optimized genetic algorithm is proposed to prove the effectiveness of the designed model.

(3) Compared with the traditional e-commerce dual channel supply chain coordination compensation model, the experimental results show that the model can effectively realize the adjustment and compensation of e-commerce dual channel supply chain coordination.

However, the compensation strategy mechanism designed in this paper is designed under the conditions of demand determination and information symmetry. The situation of demand uncertainty and information asymmetry will be the direction of further research.

\section{REFERENCES}

[1] B. Yan, Z. Chen, X. N. Wang and Z. J. Jin, "Influence of logistic service level on multichannel decision of a two-echelon supply chain", International Journal of Production Research, vol. 58, no. 11, pp. 3304-3329, 2020.

[2] G. Y. Xu and H. G. Qiu, "Pricing and distribution strategies in a dual-channel supply chain", International Journal of Information Systems and Supply Chain Management, vol. 13, no. 3, pp. 23-37, 2020.

[3] M. Noori-Daryan and A. A Taleizadeh, "Optimizing pricing and ordering strategies in a three-level supply chain under return policy", Journal of Industrial Engineering International, vol. 15, no. 1, pp. 73-80, 2019.

[4] Z. Zhang, M. H. Chen, H. Ke and A. S. Vinhas, "The influences of channel subsidy on consumers in a dual-channel supply chain", Soft Computing: A Fusion 
of Foundations, Methodologies and Applications, vol. 24, no. 2, pp. 5101-5110, 2020.

[5] C. W. Dong, Y. Liu and T. N. Chi, "Quantity leadership for a dual-channel supply chain with retail service", Asia-Pacific Journal of Operational Research, vol. 37, no. 2, pp. 32-40, 2020.

[6] R. Hammami, Y. Frein and A. S. Albana, "Delivery time quotation and pricing in two-stage supply chains: Centralized decision-making with global and local managerial approaches", European Journal of Operational Research, vol. 286, no. 1, pp. 164-177, 2020.

[7] S. D. Ekşioğlu, B. Gulcan, M. Roni and S. Mason, "A stochastic biomass blending problem in decentralized supply chains", Naval Research Logistics, vol. 68, no. 4, pp. 434-453, 2021.

[8] M. Johari and S. M. Hosseini-Motlagh, "Coordination contract for a competitive pharmaceutical supply chain considering corporate social responsibility and pricing decisions", RAIRO-Operations Research, vol. 54, no. 5, pp. 1515-1535, 2020.

[9] M. Eghbali-Zarch, A. A. Taleizadeh and R. Tavakkoli-Moghaddam, "Pricing decisions in a multiechelon supply chain under a bundling strategy", International Transactions in Operational Research, vol. 26, no. 6, pp. 2096-2128, 2019.

[10] L. Dupont, C. Bernard, F. Hamdi and F. Masmoudi, "Supplier selection under risk of delivery failure: A decision-support model considering managers' risk sensitivity", International Journal of Production Research, vol. 56, no. 3, pp. 1054-1069, 2018.

[11]C. Kogler and P. Rauch, "A discrete-event simulation model to test multimodal strategies for a greener and more resilient wood supply", Canadian Journal of Forest Research, vol. 2019, no. 49, pp. 1298-1310, 2019.

[12]B. Karimi, A. H. Niknamfar, B. H. Gavyar, M. Barzegar and A. Mohtashami, "Multi-objective multi-facility green manufacturing closed-loop supply chain under uncertain environment", Assembly Automation, vol. 39, no. 1, pp. 58-76, 2019.

[13] A. Azimian and B. Aouni, "Supply chain management through the stochastic goal programming model", Annals of Operations Research, vol. 251, no. 1-2, pp. 351-365, 2017.

[14] A. Eydi and A. Fathi, "An integrated decision making model for supplier and carrier selection with emphasis on the environmental factors", Soft Computing, vol. 24, no. 6, pp. 4243-4258, 2020.

[15] M. El-Nemr, M. Afifi, H. Rezk and M. N. F. Ibrahim, "Finite element based overall optimization of switched reluctance motor using multi-objective genetic algorithm (NSGA-II)", Mathematics, vol. 9, no. 5, pp. 576-576, 2021.

[16] C. A. Pramono, A. H. Manurung, P. Heriyati and W. Kosasih, "Analysis of the influence of entrepreneurship capability, agility, business transformation, opportunity on start-up behavior in e-commerce companies in Indonesia during the Covid 19 pandemic", WSEAS Transactions on Business and Economics, vol. 18, pp. 1103-1112, 2021.

[17] V. Y. Chernova and E. A. Degtereva. "Forecast of development of the dual-use industrial products market", WSEAS Transactions on Business and Economics, vol. 17, pp. 987-992, 2020.

[18]A. G. Eldar, "Problems of financial and investment support modelling of the regional social and economic development", WSEAS Transactions on Business and Economics, vol. 17, pp. 741-752, 2020.

[19] M. Van den Bossche, C. Noguera and J. Goniakowski, "Understanding the structural diversity of freestanding $\mathrm{Al}_{2} \mathrm{O}_{3}$ ultrathin films through a DFTB-aided genetic algorithm", Nanoscale, vol. 12, no. 10, 6153-6163, 2020.

[20]D. A. Sitar-Tut, D. Mican and O. Z. MRS, "Managerial recommender system for electronic commerce based on Onicescu method and Zipf's law", Information Technology and Management, vol. 21, no. 2, pp. 131-143, 2020.

[21]T. Kawai and S. Imada, "Energy distribution of small-scale flares derived using a genetic algorithm", The Astrophysical Journal, vol. 906, no. 1, pp. 2-10, 2021.

[22]Z. G. Li, L. Stan, D. A. Czaplewski, X. D. Yang and J. Gao, "Broadband infrared binary-pattern metasurface absorbers with micro-genetic algorithm optimization", Optics Letters, vol. 44, no. 1, pp. 114-117, 2019.

[23]B. Fahimnia, J. Sarkis, A. Gunasekaran and R. Farahani, "Decision models for sustainable supply chain design and management", Annals of Operations Research, vol. 250, no. 2, pp. 277-278, 2017.

[24] A. S. Al-Adwan, M. Alrousan, A. Al-Soud and H. Al-Yaseen, "Revealing the black box of shifting from electronic commerce to mobile commerce: The case of Jordan", Journal of Theoretical and Applied Electronic Commerce Research, vol. 14, no. 1, pp. 51-67, 2019.

\section{Creative Commons Attribution License 4.0 (Attribution 4.0 International, CC BY 4.0)}

This article is published under the terms of the Creative Commons Attribution License 4.0

https://creativecommons.org/licenses/by/4.0/deed.en_US 